\title{
El matrimonio místico del chamán y el animismo entre los asháninka del oriente peruano
}

El presente artículo muestra el lugar del chamán en un sistema cosmológico de carácter animista como el de los asháninka. A partir de su matrimonio con el espíritu del tabaco el chamán asháninka repara las relaciones entre humanos, y humanos con no humanos.

Palabras clave: Chamanismo; animismo; tierras bajas de América del Sur; asháninka; arawak preandinos.

\section{Mystique marriage of the chaman and animism among the ashaninka of Eastern Peru}

ABSTRACT

This article explains the place of the chaman in the animistic cosmological system of the ashaninka. Since his marriage with the spirit of the tobacco he is able to repair the relations between humans, and humans with not humans.

KeYwords: Chamanism; animism; lowland South America; Ashaninka; Pre-andean Arawak. 


\section{Introducción}

E n el presente artículo entendemos por chamán un tipo de especialista ritual caracterizado por la atribución por el entorno social del poder de viajar allí donde se encuentran los espíritus y que, por lo tanto, ocupa un lugar central en el intercambio y comunicación con estos. Este tipo de institución corresponde a los sistemas de representaciones de tipo animista, en que los no humanos poseen en su interioridad un principio espiritual semejante al de un ser humano (Descola 2005: 178). La figura del viaje al lugar de habitación de los espíritus es representada por estos especialistas en diferentes culturas por diferentes medios vocales (silbidos, gritos, quejidos, cambios en el tono de la voz, cánticos) y gestuales (desde la imitación mímica de animales o de la embriaguez, hasta una quietud total, cataléptica), los que constituyen signos del encuentro con los espíritus (condición a menudo descrita en la literatura etnográfica con el término de «trance») (Hamayon, 1982, 1995; Chaumeil 1998). El viaje constituiría la diferencia específica de este tipo de especialista respecto a los de las religiones de posesión, en que, por el contrario, los espíritus llegan, se alojan y animan el cuerpo del médium (de Heusch 1971). Si las relaciones con los espíritus son descritas en estos sistemas en términos de parentesco como matrimoniales o de afinidad, en el caso del chamán este es entendido como el esposo de un espíritu, mientras que, a la inversa, en los sistemas de posesión el espíritu es entendido como el esposo del médium (Hamayon 1998). La organización religiosa tradicional de los asháninka es de tipo chamánico (Elick 1969, Weiss 1975, Chevalier 1982, Rojas Zolezzi, E. 2004). Empleamos aquí el término de chamán para designar a este especialista ritual ya que aquellos de medicine man o curandero introducen un sesgo en nuestra comprensión de dicho especialista. En efecto como ha señalado Hamayon (1982) hay casos de tradiciones chamánicas en que estos especialistas sólo hacen daño y no curan.

Los asháninka constituyen un grupo de horticultores de roza, cazadores, pescadores y recolectores de la familia Arawak en su rama maipureana y se encuentran en la región de la cabecera del río Ucayali, que comprende los ríos Perené, Ene, Tambo, Pichis,
Pachitea, y la región interfluvial del Gran Pajonal. Así, el territorio tradicional de este grupo comprende un sector de la varcea del Ucayali, un sector del piedemonte oriental de los Andes y zonas de tierra firme interfluviales, siendo predominantemente colinoso. El territorio asháninka se encuentra hoy bajo un fuerte proceso de colonización por parte de campesinos quechua provenientes de los Andes centrales.

\section{El animismo asháninka}

En la mitología asháninka encontramos dos tradiciones de explicación del origen de los no humanos. En una de estas los diferentes tipos de animales son el resultado de esputos que el principio femenino lanza cuando está por dar a luz al sol engendrado en ella por luna (Rojas Zolezzi, E. 2014: 107-114; 151). En la otra de estas tradiciones todos los animales son humanos transformados por el demiurgo Awireri. Este, paseaba llevando sobre sus hombros a su nieto kiri, quien cada vez que se encontraban con humanos preguntaba a su abuelo de quienes se trataba a lo que este respondía con un nombre de animal transformándolos de esta manera en la especie señalada. Los asháninka, molestos por esto, lo envían al final del río a través de un túnel al que hacen caer a Awireri. El nieto kiri va en busca de su abuelo pero es finalmente alcanzado y muerto clavándole una estaca en la cabeza de donde sale mucha sangre la que se convierte en un lago. En esta sangre se bañan diferentes aves adquiriendo los bellos y coloridos plumajes que los distinguen como especies (Weiss 1975: 309-344; Rojas Zolezzi, E. 2014: 176-188). Se trata pues de la definición de los diferentes tipos de no humanos. La definición de algunas otras especies es atribuida a acciones del sol, como cuando este transforma a un hombre que masticaba carne humana en el pez boquichico shimá (Prochilodus sp.) (Weiss 1975). Sin embargo hay que señalar que la transformación, pianaka, de estos seres es de su fisicalidad, permaneciendo su interioridad semejante a la humana atribuyéndoles sentimientos y acciones semejantes a las de los humanos. Único ser capaz de atravesar en ambos sentidos el límite entre humanos y no humanos es el chamán en este sistema, quien podrá convertirse en jaguar (pantera onca) o colibrí (fam. Trochilidae) a voluntad. 


\section{La obtención del poder chamánico}

Se llega a la posición de chamán a través de un proceso de aprendizaje bajo la dirección de otro chamán y que lleva a una transformación (pianaka) del ser del individuo. El término que designa a este especialista es sheripiari que podemos traducir como aquel que se transforma por el tabaco (sheri, tabaco; pia- de piánaka, transformación; -ri $3^{\text {a }}$ persona masculina). La transformación se opera a través del consumo de pasta de tabaco que el chamán instructor dará al aprendiz, extracto de hojas hervidas de esta planta que recibe el nombre de "carne del tabaco" (owatsa pocharo). A esta preparación se ańadirá progresivamente el consumo de la savia de la hayapa (Brugmancia sp., toé en español regional ). Al cabo de un periodo de uno o dos meses, durante los cuales el individuo vivirá en el bosque aislado de todo contacto femenino y comiendo sólo yuca hervida y bebiendo agua de manantial, el aprendiz adquirirá la capacidad de transformarse como hemos señalado en jaguar o colibrí según necesite desplazarse por tierra o aire. En el momento en que tiene la visión de una mujer que es el espíritu femenino del tabaco, la que se convertirá en su esposa, entonces adquirirá la capacidad de transformarse en jaguar y curar a las personas enfermas. En las palabras de uno de nuestros informantes: "Le ve el jaguar allá al tabaco que es mujer, le ve ahora que viene ahora en forma de mujer. Dice: ajá el coge conocimientos y quiere ser chamán. Antiguamente era humano el tabaco. Ahora se comienza a transformar en chamán. Ahora vienen a él los enfermos» ("Iñakeroma manitsi hantá sherira koyaonatsi iñawakerora pokapake iroñaka osharo koyara. Ikantsi aháyentakotatyaro añantamentotsi ikowatsi isheripiarite irirori irohatsite yawiantakiaro. Pairani irotake atsiritatsi sheri. Ari onpoñanakiari yawianakero isheripiyate. Ironaka amenantanake mantsiyari»). ${ }^{1}$ Este matrimonio místico exige al aprendiz mantenerse célibe pues una relación con una mujer humana interferiría con su relación con el espíritu femenino del tabaco. Es este matrimonio místico el que le da al chamán la capacidad de transformarse en jaguar y le permitirá destruir a los agresores de los miembros de su grupo residencial o nampitsi, incluidos chamanes

1 Tessman a finales de los años 1920 recogió una versión de esta misma representación en la cual el espíritu del tabaco es un ser masculino, lo que consideramos un error de traducción (Tessman 1999: 51). de otros asentamientos y brujos. De otra parte, su transformación en colibrí le permite viajar al lugar de residencia del dueño de las diferentes especies de presas de caza en las alturas del asentamiento, en donde las cuida a la manera de hatos. Se trata de un ser de figura antropomorfa muy delgado con un pie doblado hacia arriba. Hay un caso femenino, el de la dueña de los pecaríes (Tayassu Tajacu) la cual posee una piel de este animal que agitará transformándose las cerdas en presas de esta especie (Weiss 1975; Rojas Zolezzi, E. 2014). El chamán viajará al lugar de habitación de este ser y le rogará que le haga entrega de presas de caza para la alimentación de los miembros de su asentamiento. Este viaje el chamán deberá realizarlo premunido de un morral grande y resistente en el que cargará las presas que le entregará el dueño de los animales hasta su comunidad.

\section{Los poderes con los que trabaja el chamán y la curación de los enfermos}

Un número de flechas invisibles son entregadas por el chamán instructor a su aprendiz y que este guarda en sus antebrazos. Estas son transmitidas de padre a hijo a lo largo de generaciones de chamanes si bien pueden ser entregadas también por un chamán a un yerno que haya sido iniciado por él. Además de estas flechas, los chamanes trabajan con seres del cosmos asháninka, sus auxiliares, que les proveen de armas ofensivas adicionales. Estos son el jaguar maniti (Pantera onca), el pez boquichico shimá (Prochilodus $s p$.), el arcoíris oye y la lluvia inkani. Cada uno de estos es la fuente de un tipo de flecha que el chamán guardará en su antebrazo. Para atacar a los agresores de sus parientes y así curarlos y cuidar a los miembros de su asentamiento el chamán asháninka realizará el gesto ikishokawakeri: este consiste en hacer un tubo con los dedos y soplar por este, lo que es entendido como el lanzamiento de una de sus flechas invisibles.

El chamán asháninka en tanto que ser poderoso es no solamente un ser benéfico sino un ser potencialmente peligroso variando el juicio de buenos /malos según la proximidad o la lejanía en términos de relaciones de parentesco entre el chamán y el hablante. El término para designar a los chamanes enemigos es amahónkari, el que corresponde también a los individuos que iniciaron el aprendizaje del conocimiento chamánico pero que no llegaron a adquirir la capaci- 
dad de curar. En otras palabras, son aquellos que sólo saben dañar. Sin embargo, en la dinámica del chamanismo las armas ofensivas son siempre necesarias en tanto que para curar a una persona se requiere neutralizar al atacante. De este modo, la noción de amahonkari se muestra pues como una categoría relativa dado que todo chamán será amahonkari para aquellos que no forman parte de su red de parentesco o su ámbito de influencia. Si el arcoíris, el pez boquichico y la lluvia son considerados auxiliares ofensivos, se atribuirá al propio chamán el trabajar con seres más conviviales como el trueno tsonkare, el colibrí tsonkiri (Fam. Trochilidae de pico recto, donador del tabaco) y la golondrina ashiwanti (Chaetura sp., donadora de las orugas y escarabajos comestibles) ${ }^{2}$.

Si hay chamanes enemigos que atacan a las familias al cuidado de un chamán, al interior del grupo de influencia de este puede haber infiltrados seres malevolentes, los matsi. Esta categoría, que traduciremos por brujo es la de aquellos quienes han sido enseñados por los seres malevolentes del bosque o kamari a hacer daño a las personas a través de la manipulación de restos de comida, cabellos o uñas enterrándolos bajo el suelo de la casa. La persona enferma verá a su atacante en sueńos y lo acusará. Otra posibilidad es que el chamán identifique al atacante a través de las visiones que le produzca la ingesta de pasta de tabaco. Los acusados son generalmente niños de ambos sexos y mujeres jóvenes. A fin de detener sus ataques,

2 Chevalier (1982:350-360, 400) reporta una lista de auxiliares adicional, obtenida de un chamán asháninka asentado en el pueblo colono de Puerto Inca sobre el río Pachitea, la cual incluye seres como «el abuelo ballena, el «Banco Muraya» o «banco del brujo», una "reinita» y una «sirena». Aunque estos fueron rechazados como falsa información por nuestros informantes habitantes de las zonas más tradicionales del territorio asháninka de los ríos Ene y Tambo pensamos que la incorporación de estos seres al sistema de representaciones vinculado al chamanismo asháninka obedece a la búsqueda de protección por poderes foráneos en un contexto en que debido a la colonización el poder en la región es detentado por los blancos y mestizos y los asháninka son empujados a una situación de marginalidad. A diferencia de las religiones de libro con estructura eclesial a cargo de la defensa de dogmas definidos y cerrados, las religiones chamánicas, son sistemas abiertos, basados en la negociación tanto con los poderes ya conocidos como con aquellos nuevos que aparecen en el horizonte, lo que las hace capaces de incorporar nuevos elementos provenientes de un flujo exterior sin perder su propia coherencia (Hell, B. 1999; Hamayon, R. 2001). Así, no es incoherente que un chamán indígena en un mundo nuevo en que los blancos y mestizos controlan la circulación del dinero, busque para sus seguidores la protección de un banquero; en un país en que los centros de poder se encuentran en la costa, es concebido como un ser poderoso el habitante mas grande del océano, la ballena; de manera similar para gente al margen del poder político busca la protección de una reina. el matsi deberá ser obligado a llorar, ya que por esta vía se cree olvidará el arte de hacer brujería. Esto se logra golpeando con plantas espinosas la piel, aplicando sustancias irritantes en los ojos o poniendo a la persona de cabeza sobre un fuego humeante.

\section{El chamán y la reparación de las relaciones con el dueño de los animales}

El chamán asháninka, como parte de su tarea de velar por el mantenimiento del equilibrio entre los humanos y otras categorías de seres del cosmos, cuida de las relaciones entre los cazadores y los dueños de las presas de caza seres que forman parte de la categoría de seres benevolentes maninkari. Una de las reglas más importantes de caza entre los asháninka es que el cazador debe mantener el cuerpo limpio de todo vestigio de contacto sexual con su esposa, es decir, de la sangre menstrual producida por ella, cuyo olor impedirá una relación adecuada con estos espíritus guardianes de las presas. Incluso una mujer que menstrua no debe alimentarse de las piezas obtenidas por el cazador. Si las presas se retiran esto es atribuido a la conducta individual de un cazador. El chamán entonces viaja transformado en colibrí hasta el lugar de habitación del dueño de los animales donde suplicará a este que le entregue presas para los cazadores de su entorno, las que traerá en su vuelo de regreso dentro de un morral fuerte y resistente con el que hará el viaje.

\section{El canto chamánico y la comunicación con las divinidades}

Los maninkari o seres benevolentes pueden visitar el lugar de habitación de los humanos en forma de pequeñas aves de colores brillantes comprendidas en la categoría de tsimeri (Weiss, G. 1975; Rojas Zolezzi, E. 2014). Al interior de esta categoría se presenta otra, la de los mensajeros de las divinidades, los colibrís de pico recto tsonkiri, portadores de los cantos performados en las ceremonias chamánicas de ingesta del psicotrópico ayahuasca (Banisteriopsis sp.) mezclada con la planta pichikipanaki (chacruna en espagnol régional, Psychotria viriidis). Estos cantos son llamados imarentaka o maretantsi. Estos canticos llegan al chamán durante su sueño psicotrópico luego de la ingestión de pasta de tabaco. En estos sueños el 
chamán recibe del colibrí tanto cantos que son considerados armas para atacar a otros chamanes enemigos pertenecientes a otros grupos locales (los que permanecen secretos) como también otros cantos de función integrativa que son performados colectivamente en la ceremonia del kamarampi o ayahuasca. Los asháninka llaman meriatiri a las alucinaciones auditivas que son experimentadas durante la ceremonia por aquellos que han bebido el psicotrópico, las cuales se considera son traídas por los maninkari. Según mis informantes esta es la fuente de todas las canciones cantadas por el chamán y escuchadas por los participantes de la celebración. Como ha señalado Weiss, los asháninka consideran que las canciones que el chamán sheripiari canta en esas ocasiones son aquellas que entonan los manínkari que han venido y que el chamán puede escuchar gracias a su acceso a los niveles más profundos de la realidad. Los chamanes asháninka se limitan a repetir estas canciones para comunicárselas a los participantes de la ceremonia, lo que constituye un medio para guiar sus alucinaciones.

Los llamados cantos imarentaka o maretantsi llegan al chamán durante el sueño psicotrópico luego de la ingestión de pasta de tabaco. Una vez obtenidos de esta manera, son integrados al ritual. A este respecto, recibiendo una lección de un chamán antiguo y experto, este me dio la explicación siguiente:

Awankiaro amishantakiaro tsonkiri iritake iritake ayótanteari

Cuando nosotros bebemos tabaco, nosotros lo conocemos a él (ashitarori tsonkiri, el dueño de los colibrís), durante la noche se sueña con el colibrí.

Ikamantemi okameka oka teowetsikashite pocharo El tabaco le dice cuando (el colibri) muere.

Paita wetsikirori chora wetsikirori tasorentsi ikantanakero

Esto es el porqué la divinidad ha creado el tabaco.

Pairani itasonkanakero hó paana ora paitari oka nowiake kempiro opita tsinkaroshi pocharo

Antiguamente la divinidad sopló (y dijo): «Lleven esto que se llama tabaco. Pónganlo en el mortero de hojas de tabaco».

Ponawakanta pisháninka arika yonkitakia "Así les asháninka tendrán el conocimiento».
Añantyairi tsonkiri irotake oká awakiaro ameistakiari tsiteniriki.

Nosotros vemos al colibri cuando tenemos sueños con aquello que bebemos.

Ameishtakero awakiaro. Chora meriatariri tsonkiri. Chora meriatariri tsonkiri.

Cuando yo bebo tabaco, yo sueño con el colibri que canta. Hay cantos de colibri.

Meriatiriririri, meriatiriririri,meriatiriririri

Onomatopeya del colibri en tren de absorber el néctar de una flor.

(Chaman José, C.N. ,Rio Tambo )

En este texto es establecida una analogía entre el chamán y el colibrí. El chaman bebe pasta de tabaco de la misma manera que el colibrí absorbe el néctar de las flores. El pasaje en el que habla de la muerte del colibrí hace referencia a las informaciones que el chamán puede recibir respecto a cómo dar la muerte a un chamán enemigo. La planta del tabaco es el medio de comunicación con un espíritu incluido en la categoría de los manínkari, el espíritu femenino del tabaco, por el cual ella transmite dichas informaciones al chamán sheripiari

Un ejemplo de los cantos de las ceremonias de ayahuasca es el siguiente:

Kenkenkeyo kenkenkeekee.

Kenkenkeyo kenkenkeekee.

Otironkanichempiki okarachempikintani.

El retorno de las cimas de los cerros y el cumplimiento.

Kenkenkeya kenkenkeke.

Ipotsonapatikiti otsitirikosatini.

Todo toma un color rojizo en el fin del mundo. Kenkenkeya kenkenke.

Yaratinkaporeeati pawiyatacharintani. okarachempikintani kowehachempikintani.

El rayo monta a las cimas de los cerros, el comienza en el final del río Cubeja sobre las cimas de los cerros.

Kenkenkeya kenkenke.

Kitamaropatikiti antyarohanisatini. 
Todo deviene blanco en la aldea de aquellos que habitan el río más grande.

Yowetsikawankotaro tsirentsipankosatini

kapashipankosatini.

Ellos construyen las casas de hoja de palmera tsirentsi (Chamaedorea sp.) y

kapashi (Euterpe precatoria).

Ipotsonapatikiti kapashipankosatini.

Las casas de hoja de palmera kapashi toman un color rojizo.

Kenkenkeya kenkenke.

Ipotsonapatiquitinta otsitirikosatininta.

El lugar donde termina el río donde ellos habitan toma un color rojizo.

Kenkenkeyaka kenkenke.

Ipotsonapatikiyanake otsitirikosatininta.

El lugar donde termina el río donde ellos habitan toma un color rojizo.

Kenkeya kenkekenke.

Kitamaropatikitinta pashiniyatosatininta okarachempikinta pashiniyatochempikinta El otro mundo donde termina el otro mundo toma un color blanco.

Kenkenkeya kenkekenke.

Kitamaropatikitinta antyaronkanisatininta okarachempikintanita.

Todo toma un color blanco en la aldea del río más grande donde termina el mundo.

Kenkenkeya kenkenkeyaka kenkenkeke.

Yapatomirinkantanakaronta shinonkakacharinta

karanpawantinisatinta

karanpawantinichempikitinta.

Ellos se reunen siempre los espiritus del mundo entero y aquellos que conocen el

mundo de los espíritus.

Kenkenkeyaka kenkenkekenke.

En este canto, se nos habla de la vida de los espíritus en otsitiriko, el lugar donde termina el río, lugar donde emana un color rojizo, referencia a los colores del sol al final del día. En otsitiriko, el fin del mun- do, habitan los espiritus ishire de los asháninka en la comunidad local más grande que puede existir y que han devenido ancestros. La vida de los ancestros es descrita como semejante a la de los humanos de esta tierra habitando en aldeas en casas con techos de palmera, pero se considera que viven sin guerra ni enfermedades. Estos espíritus ancestrales no retienen ni el nombre ni las características individuales, fundiéndose en el conjunto de los espíritus que habitan otsitiriko bajo la forma de luces blancas y rojas. Según mis informantes, las luces rojas son las almas de los asháninka que han muerto por heridas producidas por los guerreros; las luces blancas son las almas de aquellos que devinieron sabios. Los únicos con capacidad para viajar a estos lugares son los chamanes.

Si los asháninka reciben la visita de los maninka$r i$, las grandes divinidades Tasorentsi se mantienen alejadas. En una época al inicio del tiempo estas vivían sobre la tierra con los asháninka pero debido a una situación de guerra permanente y faltas como el incesto se fueron al cielo. Así, el sol decidió irse a lo alto cuando descubrió una relación incestuosa entre un hijo suyo y su esposa (Anderson 1986, III: 138-141). Asimismo, los asháninka antiguamente conocían el camino para llegar donde habita la divinidad Pachákama, productora y donadora de los utensilios de metal pero este conocimiento se ha perdido (Varese 1973:309-311; Rojas Zolezzi, E. 2004 Vol.I: 215-216). Los asháninka guardan la esperanza de una nueva reunión y expresan su tristeza por la separación en cantos maretantsi como el siguiente:

Pasanketya pasanketya pasankearitiari tasorentsi tasorentsisanori

Siente la presencia siente la presencia del poderoso del poderoso.

\section{Wishirentsisanori ihokimatanake}

Nosotros estamos muy tristes porque él nos ha abandonado.

Impoi irantanahiari tasorentsisanori onimoheetanake iránahe inkiteki iriyori

Mas tarde él nos llevará el verdadero poderoso, nosotros estamos contentos porque él nos llevará al cielo.

Antiguamente, cuando los guerreros mataban a los asháninka no importa dónde, en un estado de violen- 
cia permanente al interior de esta población, los guerreros avispa sani (Genero Polistes), puerco espín tontori (Coendu bicolor) y el perezoso soroni (Choloepus hoffmanni) intentaron llevar la guerra al cielo. Para evitar esto las divinidades una vez llegados al cielo cortaron la liana para evitar que sus perseguidores suban cayendo estos por tierra. La avispa se convirtió en el insecto que hoy vemos al atravesarse con una de sus flechas; el puercoespín se atravesó con sus propias flechas y se transformó en el ser lleno de púas que hoy vemos. El perezoso que era experto en producir sogas para hacer trampas se enredó en éstas que el llevaba consigo volviéndose el peludo ser que hoy vemos. La canción aquí presentada expresa el sentimiento de tristeza por la lejanía de las divinidades y la esperanza de que en el futuro divinidades y humanos vuelvan a estar juntos.

\section{Alucinaciones visuales y expresiones estéticas}

Las alucinaciones auditivas son acompañadas en la celebración del ayahuasca por aquellas visuales o fosfenos. Estas son figuras geométricas y combinaciones de colores que se dice son la visión de los espíritus visitantes en su verdadera forma que corresponde a los diseños geométricos representados por las mujeres en los tejidos. Señalamos a continuación los ejemplos más comunes.

Entre los seres representados en diseños geométricos tenemos los siguientes:

- Escama de pez kipaori o kipaoritsapa (Parodon $s p$.): diseño formado por un conjunto de líneas rectas discontinuas intercaladas con líneas continuas; hilo oscuro sobre fondo blanco.

- Arco iris: diseño de líneas negras paralelas continuas sobre fondo blanco denominado pitsitsari; las líneas representarían las bandas de colores del arco iris.

- Cola del pez boquichico (Prochilodus sp.) shimashiriki: diseño constituido por dos triángulos isóseles unidos por los ángulos opuestos a sus bases, con sus superficies coloreadas en color oscuro; los dos triángulos representarían las dos puntas de la cola.

- Loros: diseño en forma de rombos sucesivos denominado ponkaro; estos representan la forma del pico visto de frente.
- Cerro otishi: línea en zig-zag de cuatro segmentos que representa alturas sobre el terreno.

Algunos de los diseños constituidos por combinaciones de hilos de colores formando largas bandas son los siguientes:

- Gusano comestible shoopa (suborden Ditrysia): combinación de morado rojizo con negro.

- Gusano comestible kariyeni: combinación de hilos amarillos y negros formando franjas paralelas de ambos colores.

- Gusano comestible kirichenki: combinación de hilos negros y amarillo rojizo o anaranjado.

- Hormiga kiáwori: combinación de hilos rojos y negros.

- Escama de pescado taakirenchi: combinación de hilos blancos, negros, amarillos y rojos.

Aún cuando la secuencia en que se disponen los hilos varía de tejedora a tejedora, las combinaciones de colores constituyen una constante. Según hemos podido observar, los colores elegidos para la representación textil del gusano comestible shoopa tratan de acercarse a los de este insecto, por lo que podemos considerar que en este caso se busca también una relación de semejanza con lo representado, que definiría a estos diseños basados en la combinación de hilos de colores también como íconos.

Los asháninka cuelgan de las bandas para portar bebés huesos en que son tallados iconos que representan diferentes especies de animales. Estos diseños también son vistos en la ceremonia del ayahuasca. Se produce una suerte de retroalimentación entre los diseńos geométricos observados de esta manera en la vida cotidiana desde la más tierna infancia y aquellos vistos bajo el efecto de los psicotrópicos.

\section{El chamán y los espiritus de los guerreros caidos}

Es fuertemente deseada por los miembros de una expedición guerrera la presencia entre ellos de un chamán, ya que se cree que los parientes caídos en acción pueden convertirse en fantasmas que golpearán a sus familiares produciéndoles enfermedades. El chamán para evitar esto llevará a cabo una ceremonia en que colocará sobre el cuerpo del caído en combate trozos de corteza de ciertos árboles sobre la cabeza, el estómago y los pies. Asimismo, un bulbo de iwenki 
(Cyperus sp.) será colocado sobre el estómago, uno por cada miembro de la partida.

\section{El caso de las chamanas}

El caso de las mujeres que se convierten en chamanas es particularmente raro en el caso asháninka. De hecho solo hemos encontrado un caso en esta población indígena. Este parecía un caso extremo para mantener el saber chamánico en el asentamiento en que encontramos este caso. El chamán de dicho grupo local había fallecido antes de transmitir todo su conocimiento a uno de sus hijos que estaba siendo enseñado para ser sheripiari. En esas circunstancias la esposa del chamán se mantuvo como depositaria de los conocimientos que le había transmitido su difunto esposo incluyendo la preparación de la bebida psicotrópica y la celebración de la ceremonia del ayahuasca la que era dirigida por el hijo que no había concluido la preparación como chamán sheripiari. Una nieta de la chamana según pudimos observar empezó a ser preparada en las tradiciones chamánicas para concertar una unión matrimonial con la familia de un chamán en otro asentamiento y así beneficiar al de la chamana de las capacidades de ese chamán para conseguir presas de caza para los residentes.

\section{El chamán y el destino de los muertos}

No existe un único destino para los muertos en el sistema de representaciones asháninka. Este dependerá del nivel de conocimientos alcanzado en vida por la persona. La gran mayoría de los asháninka, se transformarán en presas de caza al cuidado de los dueños de los animales. Esta concepción guarda relación con el hecho de que la caza juega un papel central en la subsistencia, de modo que, en este contexto, esta es la forma que asume la herencia. Otros terminarán en el estómago de Luna al caer en su trampa para peces situada río abajo. Los guerreros pueden ir a la tierra de los guerreros muertos itentari o se convertirán en gallinazos tisoni (Daptrius ater) bajo el comando del condor amempiri (Vultur gryphus). Entre los chamanes por su parte los más sabios entrarán al cielo henoki donde se sumarán a los maninkari o seres benevolentes que allí habitan; un chamán menos sabio se transformará en un jaguar de modo permanente que velará por los miembros de su nampitsi o grupo residencial. El cuerpo del chamán es abandonado en la altura que domina el grupo residencial, lugar que desde ese momento se convierte en un lugar prohibido para todos. Si el chamán sheripiari puede convertirse a voluntad al anochecer en jaguar, se cree que las chamanas sólo se transformarán en jaguares luego de su fallecimiento. Estas representaciones guardan coherencia con la concepción animista de la cosmología asháninka.

\section{Discusión}

Las acciones del chamán se encuentran ubicadas tanto en la explotación de los no humanos en las actividades productivas como en la salvaguarda de la integridad de los miembros del grupo local y las relaciones con los espíritus ancestrales. Incluso guardan una relación con las expresiones estéticas de esta sociedad. En este sentido se encuentran en todos los aspectos de la vida social lo que hace al chamanismo asháninka un hecho social total.

En la relación con los espíritus ancestrales $m a-$ ninkari, el chamán constituye ante todo un comunicador entre los humanos y estos. Parte de esa función tiene que ver con la relación con las presas de caza por lo que su intervención es considerada parte de los factores de producción. A la vez, en tanto que comunicador, debe regular las relaciones con los seres malevolentes kamari, evitando así la transformación de los humanos en nuevos seres kamari, lo que pondría en peligro el equilibrio de la sociedad y del cosmos.

Weiss (1973) interpretó estos roles del chamán en términos evolucionistas como una posición intermedia entre la figura del chamán y la del sacerdote. Nosotros por nuestra parte pensamos que estos se encuentran plenamente en una dinámica propia a los sistemas chamánicos de comunicación entre humanos, no humanos y espíritus que consiste en la negociación y el juego con los poderes ya conocidos o nuevos presentes en el cosmos (Hamayon, R. 1993; Hell, B. 1999).

En su actuar de salvaguarda de la integridad de los humanos que forman parte de su grupo de influencia, las acciones del chamán pueden coincidir con las de los líderes locales y guerreros. Sin embargo, si bien puede haber conjunción de objetivos, sólo el chamán tiene la capacidad de representar al grupo de 
los humanos ante los espíritus, ámbito específico del chamanismo (Descola y Lory 1982: 105-106).

El chamanismo asháninka no está excento de los cambios que la colonización y la expansión del mercado en la región imprimen a la sociedad asháninka lentamente. Asi, tanto Chevalier (1982) como Lenaertz (2004) nos muestran casos de chamanes que se dedican únicamente a realizar curaciones, dejando de lado las funciones de reparadores de las relaciones con los dueños de las presas de caza y comunicadores con los espíritus ancestrales. Se trata entonces de un cambio por disociación de funciones.

\section{Conclusiones}

A través de esta revisión de los diferentes aspectos del chamanismo asháninka encontramos que es la alianza matrimonial del chamán con el espíritu femenino del tabaco la que permite la comunicación de los humanos con las divinidades y los espíritus. Así, corresponde al modelo de intermediación entre las fuerzas del cosmos y los hombres en la búsqueda permanente del equilibrio homeostático enunciado por Reichel- Dollmatoff (1975). Esta cosmología por otra parte corresponde con el modelo que Descola ha denominado animismo reformulando un concepto de larga data en la antropología y que hace referencia a un sistema de representaciones en que se atribuye a los no humanos una interioridad semejante a la de los humanos y una fisicalidad distinta. Finalmente podemos decir que el caso asháninka corrobora la afirmación de este autor de que el chaman en una cosmología animista es el único capaz de «atravesar las fronteras de... compartimentación jerárquica y de trascender la fragmentación ontológica» entre humanos y no humanos (Descola 2005: 507).

\section{Bibliografía}

Anderson, Jaime (1986). Cuentos folklóricos de los asháninka. CALAP-ILV, Yarinacocha, T-III.

AugÉ, Marc (1982). Génie du paganisme. Éditions Gallimard, Paris.

BAER, Gerhart (1992). «The one intoxicated by tobacco. Matsiguenga Shamanism». En: Matteson,J.L. y Baer, G. (ed) Portals of Power. Shamanism in South America.
Alburquerque, University of New Mexico Press: 79-100.

Chaumeil,Jean-Pierre (1998). Ver, saber, poder. Chamanismo de los Yagua de la Amazonía peruana. IFEA, CAAAP, CAEA-CONICET, Lima.

Chevalier,J. (1982) Civilization and the Stolen Gift: Capital, kin and cult in Eastern Peru. Toronto University.

Crépeau, Robert (ed.) (1988). Chamanismes des Amériques, Recherches amérindiennes au Québec. Numéro spécial, 28, Montreal.

Descola, Philippe (2005). Par- dela nature et culture. Éditions Gallimard, Paris.

Descola, Ph, y Lory, J-L. (1982). «Les guerriers de l'invisible. Sociologie comparative de l'agression chamanique en Papouasie Nouvelle-Guinée et en Haute Amazonie (Achuar)». En: L'Ethnographie (Paris) $\mathrm{n}^{\circ}$ 87-88:85-109.

Eliade, Mircea (1968) Le chamanisme et les techniques archaique de l'extase. Payot, Paris.

EuIck, John (1969) An ethnography of the Pichis Valley Campas of Eastern Peru. Ph D Dissertation, University of California.

Hamayon, Roberte (1982) «Des chamanes au chamanisme». En: Voyages chamaniques deux, L'Ethnographie (Paris) nº7-88:13-47.

Hamayon, Roberte (1990) La chasse á l'ame. Esquisse d'une theorie du chamanisme á partir d'exemples sibériens. Société d'ethnologie, Nanterre.

Hamayon, Roberte (1993) »Le «jeu» forme élementaire du ritual», En: Thines, Georges y de Heusch, Luc (eds.) Rites et Ritualisation. Paris, Institut Interdisciplinaire d'Études Epistémologiques: 65-100.

HAMAYON, Roberte (1995) "Le chamanisme sibérienne, reflexión sur un médium», La Recherche (Paris), 275: 416-422.

HamaYon, Roberte (1998) «Le sens de l'alliance religieuse. «Mari» d'esprit, «femme» de dieu». En: Anthropologie et sociétés (Paris) $\mathrm{n}^{\circ} 22$ (2): 25-48.

Hamayon, Roberte (2001) «Shamanism : symbolic system, human capability and western ideology». En: Hamayon, Roberte y Francfort, Henri-Paul (eds.) The concept of Shamanism: uses and abuses. Budapest, Akademiai Kaidó (Bibliotheca Shamanistica X), 408 pp.

Hell, Bertrand (1999). Les maitres du désordre. Possession \& Chamanisme. Flammarion, Paris.

Heusch, Luc de (1971). Pourquoi l'épouser? Éditions Gallimard, Paris. 
Hoppal, M. y Sadovszky, O.J. (eds.) (1989). Shamanism: past and present. Budapest-Los Angeles/Fullerton, Istor Books. Dos volúmenes.

Hvalkof, Soren y Veber, Hanne (2005). «Los Asháninka del Gran Pajonal». En: Santos, F. y Barclay, F. (eds.) Guia etnográfica de la Alta Amazonia, Vol. 5, IFEASmithsonian Tropical Research Institute, Lima, pp.75-279

LenaertZ, Marc (2004). Anthropologie des Indiens Ashéninka d'Amazonie. L'Harmattan, Paris.

MÉtraux, Alfred (1967). Religions et magies indigennes d'Amerique du Sud. Éditions Gallimard, Paris.

Oosten, Jarich (1989). «Theoretical problems in the study of Inuit shamanism» En: Hoppal, M y Sadovsky, O.J. (eds.): 331-340.

Perrin, Michel (1992). Les Practiciens du reve. Un exemple du chamanisme. PUF, Paris.

Perrin, Michel (1995). Le chamanisme. PUF, Paris.

Reichel-Dolmatoff, Gerardo (1975). The shaman and the jaguar. A study of narcotic drugs among the Indians of Colombia. Temple University Press, Philadelphia.
Rojas Zolezzi, Enrique (2004). Mythes de la création du monde, représentations du gibier et des plantes cultivées et défnition de lórdre social traditionnel chez les Campa Ashaninka de l'Orient péruvien. These de Docteur en Anthropologie Social, EHESS, Paris.

Rojas Zolezzi, Enrique (2014). El morral del colibrí. Mitología, chamanismo y ecología simbólica entre los asháninka del oriente peruano. Editorial Horizonte, Lima.

Tessman, Gunther (1999). Los indígenas del Perú Nororiental, Abya-Yala, Quito.

Vitebsкi, Piers (1995). Les Chamanes. Albin Michel, Paris. WeIss, Gerald (1973). «Shamanism and priesthood in the light of the Campa Ayahuasca Ceremony». En: Harner, M. (ed) Hallucinogens and Shamanism, Oxford University Press, pp. 40-47.

WeIss, Gerald (1975). Campa Cosmology. The world of a forest tribe in South America. Anthropological papers of the Museum of Natural History, New York.

Weiss, Gerald (2005). «Campa Ribereños». En: Santos, F. y Barclay, F. Guía Etnográfica de la Alta Amazonia, Vol. 5, IFEA-Smithsonian Tropical Research Institute, Lima, pp. 1-74. 